\title{
Конечные группы с почти большим неприводимым характером
}

Никитина А.А., студент, Северо-Восточный федеральный университет им. М.К. Аммосова, 2. Якутск

E-mail: anjelikanikit97@mail.ru

Научный руководитель: к.ф.-м.н, доцент Поисеева С.С.

Пусть G - неединичная, конечная группа с неприводимым комплексным характером $\Theta$, степени $\Theta(1)$. В общем случае, порядок конечной группы существенно больше квадрата степени любого ее, неприводимого характера. Согласно соотношениям ортогональности, для неприводимых характеров, сумма квадратов степеней этих характеров равна порядку группы G. В частности, $\Theta(1)^{2}<|G|$.

Однако, ранее в [1, стр. 484] авторами были изучены конечные группы с большим неприводимым характером, которые были названы LC $(\Theta)$-группами, т.е. конечные неединичные группы $G$, обладающие неприводимым характером $\Theta$, таким что $2 \Theta(1)^{2} \geq$ $|G|$ и порядка больше двух.

Напомним, что в [2, стр. 81] было замечено, что в «Атласе простых конечных групп» [3] нет таких групп, у которых степень комплексного, неприводимого характера $\Theta$, удовлетворяла бы условию $2 \Theta(1)^{2} \geq|G|$, однако были указаны 8 простых групп удовлетворяющих условию $\Theta(1)^{2} \geq|G|$, при с $<3$ :

Таким неприводимым характером $\Theta$ степени $\Theta(1)=8$, при с $<2,7$ обладает группа $\mathrm{L}_{3}(2)$ порядка 168. При с $<2,6$ спорадическая группа Томпсона $T h=$ $F_{-}(3 \mid 3)$ обладает неприводимым характером степени $\Theta(1)=190373976$ т.e. $|\mathrm{Th}|<$ $2,51 \Theta(1)^{2}$. Знакопеременные группы $A_{5}, A_{7}$ при с $<2,5$ имеют неприводимый характер степени 5 и 35 соответственно. При с $<3$ четыре группы Матье $\mathrm{M}_{11}, \mathrm{M}_{22}, \mathrm{M}_{23}, \mathrm{M}_{24}$ имеют неприводимый характер $\Theta$ степени $\Theta(1)$ равный соответственно 55, 385, 2024, 10395.

Цель настоящей статьи - изучение конечных групп, удовлетворяющих условию $\mathrm{c} \Theta(1)^{2} \geq|\mathrm{G}|$, при $2<\mathrm{c} \leq 3$ :

Определение. Конечную группу G, порядка больше трех, обладающую таким неприводимым характером $\theta$ таким, что $3 \theta(1)^{2} \geq|G|>2 \theta(1)^{2}$ будем называть ALC( $\left.\theta\right)$ группой (от английского “Almostlargecharacter" - группой с почти большим неприводимым характером).

Ниже будут сформулированы результаты об ALC ( $\theta)$-группах с неприводимым характером $\theta$ степени р.

Теорема. Пусть $\mathrm{G}$ - ALC $(\theta)$-группа, у которой $\theta(1)=p$, где $\mathrm{p}$ - простое. Тогда возможны:

1. $|G|=12, p=2$;

2. $|G|=27, p=3$;

3. $G \cong A_{5}$;

4. $G \cong C_{2} \times R$, где $\mathrm{R}-$ Фробениуса группа порядка $\mathrm{p}(\mathrm{p}+1)$ и $\mathrm{p}=2^{\mathrm{b}}-1$ - простое число Мерсенна, $b \in \mathbb{N}$; 
5. $G \cong Q \rtimes C_{p}$, где $|Q|=2 p+1$ - простое число.

Для дополнительных вычислений использована система GAP [4] компьютерной алгебры. С помощью GAP составлен список конечных групп, с почти большим неприводимым характером, до 3403 порядка, а также построены таблицы характеров некоторых данных групп.

Ниже будут представлены таблицы характеров ALC $(\theta)$-групп с неприводимым характером $\theta$ степени р.

$$
\begin{aligned}
& 1 . G=C_{3} \rtimes C_{4} \\
& |G|=12=2^{2} \cdot 3, \\
& \theta(1)=2, \text { гдеа }=-\varepsilon_{4}=-\sqrt{-1}=-i
\end{aligned}
$$

\begin{tabular}{|c|c|c|c|c|c|c|}
\hline & $|G|$ & 4 & $|G|$ & 6 & 4 & 6 \\
& $1 \mathrm{~A}$ & $4 \mathrm{~A}$ & $2 \mathrm{~A}$ & $3 \mathrm{~A}$ & $4 \mathrm{~B}$ & $6 \mathrm{~A}$ \\
\hline $\mathrm{x}_{1}$ & 1 & 1 & 1 & 1 & 1 & 1 \\
\hline $\mathrm{x}_{2}$ & 1 & -1 & 1 & 1 & -1 & 1 \\
\hline $\mathrm{x}_{3}$ & 1 & $\mathrm{a}$ & -1 & 1 & $-\mathrm{a}$ & -1 \\
\hline $\mathrm{x}_{4}$ & 1 & $-\mathrm{a}$ & -1 & 1 & $\mathrm{a}$ & -1 \\
\hline $\mathrm{x}_{5}$ & 2 & 0 & -2 & -1 & 0 & 1 \\
\hline $\mathrm{x}_{6}$ & 2 & 0 & 2 & -1 & 0 & -1 \\
\hline
\end{tabular}

Таблица №1

2. $G=C_{7} \rtimes C_{3}$

$|G|=21=3 \cdot 7$,

$\theta(1)=3$, где $\mathrm{a}=\varepsilon_{3}^{2}=\frac{-1-\sqrt{-3}}{2}, b=\varepsilon_{7}+\varepsilon_{7}^{2}+\varepsilon_{7}^{4}=\frac{-1-\sqrt{-7}}{2}$,

\begin{tabular}{|c|c|c|c|c|c|}
\hline & $|\mathrm{G}|$ & 3 & 7 & 3 & 7 \\
& $1 \mathrm{~A}$ & $3 \mathrm{~A}$ & $7 \mathrm{~A}$ & $3 \mathrm{~B}$ & $7 \mathrm{~B}$ \\
\hline $\mathrm{x}_{1}$ & 1 & 1 & 1 & 1 & 1 \\
\hline $\mathrm{x}_{2}$ & 1 & $\mathrm{a}$ & $\mathrm{a}$ & $\overline{\mathrm{a}}$ & 1 \\
\hline $\mathrm{x}_{3}$ & 1 & $\overline{\mathrm{a}}$ & 1 & $\mathrm{a}$ & 1 \\
\hline $\mathrm{x}_{4}$ & 3 & 0 & $b$ & 0 & $\bar{b}$ \\
\hline $\mathrm{x}_{5}$ & 3 & 0 & $\bar{b}$ & 0 & $b$ \\
\hline
\end{tabular}

3. $G=C_{2} \times A_{4}$

$|G|=24=2^{3} \cdot 3$,

$\theta(1)=3$, где $\mathrm{a}=\varepsilon_{3}^{2}=\frac{-1-\sqrt{-3}}{2}$,

\begin{tabular}{|c|c|c|c|c|c|c|c|c|}
\hline & $|\mathrm{G}|$ & $|\mathrm{G}|$ & 6 & 8 & 6 & 8 & 6 & 6 \\
& $1 \mathrm{~A}$ & $2 \mathrm{~A}$ & $3 \mathrm{~A}$ & $2 \mathrm{~B}$ & $6 \mathrm{~A}$ & $2 \mathrm{C}$ & $3 \mathrm{~B}$ & $6 \mathrm{~B}$ \\
\hline $\mathrm{x}_{1}$ & 1 & 1 & 1 & 1 & 1 & 1 & 1 & 1 \\
\hline $\mathrm{x}_{2}$ & 1 & -1 & 1 & 1 & -1 & -1 & 1 & -1 \\
\hline $\mathrm{x}_{3}$ & 1 & -1 & $\mathrm{a}$ & 1 & $-\mathrm{a}$ & -1 & $\overline{\mathrm{a}}$ & $-\bar{a}$ \\
\hline
\end{tabular}

Таблица №3 


\begin{tabular}{|c|c|c|c|c|c|c|c|c|}
\hline $\mathrm{x}_{4}$ & 1 & -1 & $\bar{a}$ & 1 & $-\bar{a}$ & -1 & $a$ & $-a$ \\
\hline $\mathrm{x}_{5}$ & 1 & 1 & $a$ & 1 & $a$ & 1 & $\bar{a}$ & $\bar{a}$ \\
\hline $\mathrm{x}_{6}$ & 1 & 1 & $\bar{a}$ & 1 & $\bar{a}$ & 1 & $a$ & $a$ \\
\hline $\mathrm{x}_{7}$ & 3 & -3 & 0 & -1 & 0 & 1 & 0 & 0 \\
\hline $\mathrm{x}_{8}$ & 3 & -3 & 0 & -1 & 0 & -1 & 0 & 0 \\
\hline
\end{tabular}

4. $G=S_{4}$

$|G|=24=2^{3} \cdot 3$,

$\theta(1)=3$.

\begin{tabular}{|c|c|c|c|c|c|}
\hline & $|\mathrm{G}|$ & 4 & 3 & 8 & 4 \\
& $1 \mathrm{~A}$ & $2 \mathrm{~A}$ & $3 \mathrm{~A}$ & $2 \mathrm{~B}$ & $4 \mathrm{~A}$ \\
\hline $\mathrm{x}_{1}$ & 1 & 1 & 1 & 1 & 1 \\
\hline $\mathrm{x}_{2}$ & 1 & -1 & 1 & 1 & -1 \\
\hline $\mathrm{x}_{3}$ & 2 & 0 & -1 & 2 & 0 \\
\hline $\mathrm{x}_{4}$ & 3 & -1 & 0 & -1 & 1 \\
\hline $\mathrm{x}_{5}$ & 3 & 1 & 0 & -1 & -1 \\
\hline
\end{tabular}

Таблица №4

5. $G=S L(2,3)$

$|G|=24=2^{3} \cdot 3$,

$\theta(1)=3$, где $a=\varepsilon_{3}^{2}=\frac{-1-\sqrt{-3}}{2}$,

\begin{tabular}{|c|c|c|c|c|c|c|c|}
\hline & $|G|$ & 6 & 4 & $|G|$ & 6 & 6 & 6 \\
& $1 \mathrm{~A}$ & $3 \mathrm{~A}$ & $4 \mathrm{~A}$ & $2 \mathrm{~A}$ & $3 \mathrm{~B}$ & $6 \mathrm{~A}$ & $6 \mathrm{~B}$ \\
\hline $\mathrm{x}_{1}$ & 1 & 1 & 1 & 1 & 1 & 1 & 1 \\
\hline $\mathrm{x}_{2}$ & 1 & $a$ & 1 & 1 & $\bar{a}$ & $a$ & $\bar{a}$ \\
\hline $\mathrm{x}_{3}$ & 1 & $\bar{a}$ & 1 & 1 & $a$ & $\bar{a}$ & $a$ \\
\hline $\mathrm{x}_{4}$ & 2 & -1 & 0 & -2 & -1 & 1 & 1 \\
\hline $\mathrm{x}_{5}$ & 2 & $-\bar{a}$ & 0 & -2 & $-a$ & $\bar{b}$ & $a$ \\
\hline $\mathrm{x}_{6}$ & 2 & $-a$ & 0 & -2 & $-\bar{a}$ & $a$ & $\bar{a}$ \\
\hline $\mathrm{x}_{7}$ & 3 & 0 & -1 & 3 & 0 & 0 & 0 \\
\hline
\end{tabular}

Таблица №5

6. $G=\left(C_{3} \times C_{3}\right) \rtimes C_{3}$

$|G|=27=3^{3}$,

$\theta(1)=3$, где $a=\varepsilon_{3}^{2}=\frac{-1-\sqrt{-3}}{2}, b=3 \varepsilon_{3}^{2}=\frac{-3-3 \sqrt{-3}}{2}$.

Таблица №6:

\begin{tabular}{|c|c|c|c|c|c|c|c|c|c|c|c|}
\hline & $|\mathrm{G}|$ & 9 & 9 & $|\mathrm{G}|$ & 9 & 9 & 9 & $|\mathrm{G}|$ & 9 & 9 & 9 \\
& $1 \mathrm{~A}$ & $3 \mathrm{~A}$ & $3 \mathrm{~B}$ & $3 \mathrm{C}$ & $3 \mathrm{D}$ & $3 \mathrm{E}$ & $3 \mathrm{~F}$ & $3 \mathrm{G}$ & $3 \mathrm{H}$ & $3 \mathrm{I}$ & $3 \mathrm{~J}$ \\
\hline $\mathrm{x}_{1}$ & 1 & 1 & 1 & 1 & 1 & 1 & 1 & 1 & 1 & 1 & 1 \\
\hline $\mathrm{x}_{2}$ & 1 & 1 & $a$ & 1 & 1 & $a$ & $\bar{a}$ & 1 & $a$ & $\bar{a}$ & $\bar{a}$ \\
\hline $\mathrm{x}_{3}$ & 1 & 1 & $\bar{a}$ & 1 & 1 & $\bar{a}$ & $a$ & 1 & $\bar{a}$ & $a$ & $a$ \\
\hline $\mathrm{x}_{4}$ & 1 & $a$ & 1 & 1 & $\bar{a}$ & $a$ & 1 & 1 & $\bar{a}$ & $a$ & $\bar{a}$ \\
\hline
\end{tabular}




\begin{tabular}{|l|l|l|l|l|l|l|l|l|l|l|l|}
\hline $\mathrm{x}_{5}$ & 1 & $\bar{a}$ & 1 & 1 & $a$ & $\bar{a}$ & 1 & 1 & $a$ & $\bar{a}$ & $a$ \\
\hline $\mathrm{x}_{6}$ & 1 & $a$ & $a$ & 1 & $\bar{a}$ & $\bar{a}$ & $\bar{a}$ & 1 & 1 & 1 & $a$ \\
\hline $\mathrm{x}_{7}$ & 1 & $\bar{a}$ & $\bar{a}$ & 1 & $a$ & $a$ & $a$ & 1 & 1 & 1 & $\bar{a}$ \\
\hline $\mathrm{x}_{8}$ & 1 & $a$ & $\bar{a}$ & 1 & $\bar{a}$ & 1 & $a$ & 1 & $a$ & $\bar{a}$ & 1 \\
\hline $\mathrm{x}_{9}$ & 1 & $\bar{a}$ & $a$ & 1 & $a$ & 1 & $\bar{a}$ & 1 & $\bar{a}$ & $a$ & 1 \\
\hline $\mathrm{x}_{10}$ & 3 & 0 & 0 & $b$ & 0 & 0 & 0 & $\bar{b}$ & 1 & 1 & 1 \\
\hline $\mathrm{x}_{11}$ & 3 & 0 & 0 & $\bar{b}$ & 0 & 0 & 0 & $b$ & 1 & 1 & 1 \\
\hline
\end{tabular}

7. $G=C_{11} \rtimes C_{5}$

$|G|=55=5 \cdot 11$,

$\theta(1)=5$, где $a=\varepsilon_{5}^{4}, b=\varepsilon_{5}^{3}, c=\varepsilon_{11}^{2}+\varepsilon_{11}^{6}+\varepsilon_{11}^{7}+\varepsilon_{11}^{8}+\varepsilon_{11}^{10}=\frac{-1-\sqrt{-11}}{2}$.

Таблица №7:

\begin{tabular}{|c|c|c|c|c|c|c|c|}
\hline & $|\mathrm{G}|$ & 5 & 11 & 5 & 11 & 5 & 5 \\
& $1 \mathrm{~A}$ & $5 \mathrm{~A}$ & $11 \mathrm{~A}$ & $5 \mathrm{~B}$ & $11 \mathrm{~B}$ & $5 \mathrm{C}$ & $5 \mathrm{D}$ \\
\hline $\mathrm{x}_{1}$ & 1 & 1 & 1 & 1 & 1 & 1 & 1 \\
\hline $\mathrm{x}_{2}$ & 1 & $a$ & 1 & $b$ & 1 & $\bar{b}$ & $\bar{a}$ \\
\hline $\mathrm{x}_{3}$ & 1 & $b$ & 1 & $\bar{a}$ & 1 & $a$ & $\bar{b}$ \\
\hline $\mathrm{x}_{4}$ & 1 & $\bar{b}$ & 1 & $a$ & 1 & $\bar{a}$ & $b$ \\
\hline $\mathrm{x}_{5}$ & 1 & $\bar{a}$ & 1 & $\bar{b}$ & 1 & $b$ & $a$ \\
\hline $\mathrm{x}_{6}$ & 5 & 0 & $c$ & 0 & $\bar{c}$ & 0 & 0 \\
\hline $\mathrm{x}_{7}$ & 5 & 0 & $\bar{c}$ & 0 & $c$ & 0 & 0 \\
\hline
\end{tabular}

\section{Список литературы:}

1. Казарин Л.С. О конечных группах с большой степенью неприводимого характера [Текст] / Л.С. Казарин, С.С. Поисеева // Моделирование и анализ информационных систем. - Якутск, 2015. - С. 483-499.

2. Поисеева С.С. О строении конечных групп большим неприводимым характером степени $\mathrm{p}^{2} \mathrm{q}$ [Текст] / С.С. Поисеева // Математические заметки СВФУ. Якутск, 2016. -С. 81-90.

3. Convay J.H., Curtis S.P., Norton S.P., Parker R.A., Wilson R.A. Atlas of finite groups: maximal subgroups and ordinary characters for simple groups [Текст]. -Oxford: Clarendon Press, $1985-294$ c.

4. GAP - Groups, Algorithms and Programming, Version 4.9.1. [Электронный pecypc] / Aachen, St. Andrews. - 2008. - Режим доступа:http//www.gap-system.org.

5. Поисеева С.С. Группы с ограничениями н степени неприводимых характеров. [Текст]: дис. канд.физ.-мат. наук: 01.01.06: защищена 06.04.18: утв. 13.08.18 / Поисеева Саргылана Семеновна. - М., 2018. - 98 с. - Библиогр.: с.67-39. 